\title{
Cooperazione, competizione o co-opetizione? Evidenze empiriche nel settore della sanità
}

\author{
di Daniele Mascia, Francesca Pallotti, Americo Cicchetti e \\ Alessandro Lomi
}

\section{Introduzione}

È opinione ormai diffusa e condivisa che le organizzazioni non operano come attori isolati nell'ambito del proprio ambiente di riferimento; al contrario, l'azione organizzativa può essere correttamente spiegata soltanto se si considera con attenzione il legame esistente tra l'organizzazione e il proprio ambiente istituzionale e competitivo. È questo uno dei principi fondamentali che si è affermato con forza nel corso degli ultimi decenni nel campo degli studi organizzativi. Secondo una vastissima e ormai consolidata letteratura, un'ampia gamma di comportamenti e outcome organizzativi possono essere compresi andando ad analizzare la complessa struttura delle diverse tipologie di relazioni nelle quali le organizzazioni sono coinvolte ed immerse. Alcuni studi ad esempio, tra i quali spiccano quelli di Granovetter (1985) e Uzzi (1996, 1997), hanno esaminato proprio l'effetto che l'embeddedness in reti di relazioni produce sulle azioni e sui comportamenti attivati dai soggetti organizzativi, singolarmente o complessivamente considerati.

Tra le diverse relazioni che legano l'organizzazione al proprio ambiente di riferimento, quelle maggiormente investigate sia sul piano teorico che empirico riguardano i legami di collaborazione e competizione con altre organizzazioni. I primi sono stati studiati soprattutto in una prospettiva di teoria della dipendenza dalle risorse e neo-istituzionalista (Meyer e Rowan, 1977; Di Maggio e Powell, 1983). Quest'ultimo filone, in particolare, ha enfatizzato il ruolo che pressioni istituzionali e processi di legittimazione esercitano sulle organizzazioni e sulle forme organizzative all'interno di un settore attraverso processi di isomorfismo istituzionale. I secondi sono stati invece ampiamente analizzati dal filone teorico della population ecology, che ha considerato l'interdipendenza competitiva come fattore determinante per comprendere i fenomeni di sopravvivenza o di cessazione delle attività da parte di organizzazioni all'interno dei settori industriali (Hannan e Freeman, 1989). Raramente le relazioni di collaborazione e competizione interorganizzativa sono state analiz- 
zate simultaneamente. Nonostante si osservi la presenza di forti interdipendenze di natura sia collaborativa che competitiva tra le organizzazioni in numerosi settori industriali, ad oggi un esiguo numero di ricerche ha esaminato sul piano empirico le due tipologie di relazioni considerandone congiuntamente gli effetti. Riteniamo che studiare la "co-opetizione" - vale a dire la presenza simultanea di relazioni di collaborazione e competizione tra imprese - come fenomeno organizzativo possa contribuire a gettare nuova luce sulle determinanti di comportamenti e performance organizzative in settori industriali di assoluto rilievo.

Uno dei contesti caratterizzati largamente da questo dualismo è quello della sanità. Nell'ambito dei principali modelli di riferimento dei sistemi sanitari, in via generale si ritiene che i sistemi di tipo universalistico - come quello del Regno Unito e dell'Italia - siano istituzionalmente improntati alla collaborazione interorganizzativa. In questi sistemi la salute è tipicamente considerata un diritto del cittadino e lo Stato interviene programmando e coordinando l'offerta di servizi assistenziali sul territorio. Poiché la pianificazione ha come obiettivo ultimo la salute del cittadino, per la tutela di quest'ultima è prevista una forte collaborazione tra le varie organizzazioni, prevalentemente pubbliche, che compongono il sistema. Differentemente, i sistemi pluralistici - come quello americano - caratterizzati dalla forte presenza di strutture private nel mercato di offerta dei servizi, vengono normalmente indicati come sistemi a forte intensità competitiva. Il bene salute è considerato alla stregua di un bene di consumo, la cui offerta, affidata a strutture gestite prevalentemente secondo criteri di razionalità economica, è lasciata al libero gioco delle forze di mercato. Lo Stato interviene solo in minima parte nella regolazione del sistema e la singola organizzazione compie autonomamente scelte strategiche e di organizzazione delle attività. In entrambe le tipologie di sistemi, gli ultimi anni sono stati testimoni di importanti trasformazioni che hanno portato alla graduale affermazione del binomio competizione/collaborazione. Da un lato, infatti, i principali interventi di riforma avviati nel Regno Unito prima, e in Italia poi, hanno introdotto elementi volti ad accrescere i livelli di competizione tra le strutture (si pensi, ad esempio, ai meccanismi di finanziamento delle prestazioni ospedaliere, alla libertà di scelta da parte del cittadino della struttura di ricovero e cura e ai criteri di valutazione dei direttori generali, per citarne alcuni) con l'obiettivo di migliorare la performance organizzativa e di sistema (Burns e Thorpe, 1993). Dall'altro, nei sistemi diametralmente opposti, in cui i diversi provider di offerta operano in un regime di libera concorrenza, i meccanismi di funzionamento del libero mercato sono stati lentamente affiancati da elementi che spingono verso una maggiore cooperazione tra le strutture. Negli Stati Uniti, ad esempio, l'attuale funzionamento delle $\mathrm{HMO}^{1}$ e, più recente-

1. L'Health Maintenance Organization (HMO) è un'entità organizzativa (for profit o no profit) che prevede l'integrazione tra compagnie di assicurazione e provider attraverso legami che spesso prevedono accordi di natura contrattuale. L'integrazione permette di offrire coperture assicurative più convenienti indirizzando il paziente che accede al servizio verso medici e strutture consorziate. 
mente, i piani di copertura assicurativa universale, sono esempi di novità introdotte allo scopo di alimentare e favorire una maggiore collaborazione tra i diversi attori che operano nel sistema.

$\mathrm{Nel}$ complesso, questi cambiamenti sollevano alcuni importanti interrogativi che riguardano, nello specifico, il funzionamento dei sistemi sanitari. In particolare, in che misura è possibile affermare che la collaborazione e la competizione possono considerarsi come comportamenti organizzativi non alternativi? Quali fattori determinano, in contesti caratterizzati dalla competizione tra le strutture, la propensione da parte delle organizzazioni a stabilire legami di collaborazione? Per rispondere a tali domande sono stati utilizzati dati raccolti nel corso del biennio 2002-2003, relativi ad una comunità di organizzazioni ospedaliere appartenenti al servizio sanitario nazionale (SSN) che offrono servizi sanitari a più di cinque milioni di persone residenti in una delle più popolose regioni italiane. Il sistema sanitario italiano rappresenta a nostro parere un setting particolarmente ideale per questo tipo di analisi poiché, a fronte della collaborazione richiesta alle organizzazioni per assicurare la salute pubblica di tutti i cittadini, è possibile riscontrare numerosi elementi che incoraggiano comportamenti competitivi tra le strutture di offerta dei servizi che compongono il sistema (Barretta, 2007).

L'articolo è strutturato come segue. Dopo una rassegna della letteratura sui fenomeni della collaborazione e della competizione interorganizzativa, l'attenzione sarà posta sulla descrizione dei dati, delle variabili utilizzate e della metodologia di ricerca adottata. Successivamente verranno descritti i risultati dell'analisi. La sezione conclusiva del lavoro è dedicata alla discussione dei risultati e delle principali implicazioni teoriche e manageriali.

\section{Background teorico e ipotesi}

\subsection{Collaborazione, competizione e "co-opetizione"}

La letteratura ha recentemente considerato la collaborazione e la competizione come fenomeni non mutuamente esclusivi (Bengtsson e Powell, 2004), sottolineando che il concetto di "confine organizzativo" è centrale per differenziare le relazioni competitive da quelle cooperative (Oliver, 2004). La cooperazione può riguardare ogni iniziativa di collaborazione interorganizzativa tesa all'ottenimento di reciproche convenienze economiche; al contrario, attraverso la competizione, le organizzazioni intendono ottenere vantaggi competitivi sostenibili attivando comportamenti opportunistici.

In particolare, la competizione esiste in tutte quelle situazioni in cui organizzazioni diverse producono prodotti e servizi simili e lottano per la conquista della medesima nicchia di mercato (Callon, 1998). La collaborazione, invece, rappresenta un comportamento attivato da organizzazioni che lavorano congiuntamente per lo sviluppo di un prodotto o l'erogazione di un servizio, i cui ritorni economici sono distribuiti equamente fra tutti gli attori organizzati- 
vi coinvolti (Bengtsson e Kock, 2000). Da tali definizioni appare chiaro come siano proprio i confini organizzativi a funzionare da spartiacque tra questi opposti comportamenti. Mentre nel primo caso i confini organizzativi sono netti e ben delineati, nel caso delle relazioni interorganizzative essi diventano assai più labili, lasciando spazio alla condivisione di competenze, infrastrutture e interessi complementari (Powell, 1990). Nella realtà, tuttavia, raramente tali comportamenti si presentano nella loro forma pura e la letteratura suggerisce che molto più frequentemente si assiste a forme ibride di collaborazione e competizione (Bengtsson e Kock, 2000; Oliver, 2004). Per "co-opetizione" si intende proprio "the dyadic and paradoxical relationship that emerges when two firms cooperate in some activities ... and at the same time compete with each other in other activities" (Bengtsson e Kock, 2000). È un tipo di comportamento, quello della "co-opetizione", che unisce i vantaggi della condivisione di attività, da una parte, e della spinta all'efficacia e alla performance, dall'altra. Diverse teorie consentono di spiegare la "co-opetizione" come fatto organizzativo. In una prospettiva contingentista, sia la teoria dei costi di transazione che la teoria della dipendenza dalle risorse vengono comunemente utilizzate per spiegare la creazione di relazioni interorganizzative assumendo come livello di analisi la singola impresa. Mentre nel primo caso l'integrazione tra le attività di organizzazioni diverse viene intrapresa con l'obiettivo - sotto certe condizioni - di ridurre i costi di transazione (Williamson, 1985), nel secondo caso la collaborazione permette all'organizzazione di acquisire potere, influenza ed altre risorse economiche, in modo da minimizzare la propria dipendenza dall'ambiente di riferimento (Pfeffer e Salancik, 1978). Questi filoni non presentano tuttavia il fenomeno collaborativo come antitetico a quello competitivo, e si concentrano sulle particolari condizioni che danno vita alla nascita di collaborazioni interorganizzative via via crescenti e durature. Anche la teoria neoistituzionalista, nella misura in cui afferma che l'interazione dinamica tra organizzazioni condiziona la varietà di nuove forme organizzative e favorisce la legittimazione ad operare in qualunque contesto competitivo (Di Maggio e Powell, 1983), offre solide basi per ritenere che le organizzazioni possano trovarsi in situazioni di "co-opetizione".

Per quanto riguarda i fattori organizzativi che spiegano la propensione delle organizzazioni a collaborare o a competere, la letteratura fornisce motivazioni diverse. Le teorie sulla cooperazione assumono che le organizzazioni sono solitamente spinte ad instaurare rapporti di collaborazione nella misura in cui condividono interessi e competenze simili. Secondo questa prima prospettiva, dunque, la similarità - o omofilia - rispetto a determinate caratteristiche organizzative rappresenta un fattore essenziale a sostegno di rapporti di collaborazione interorganizzativa (Barley et al., 1992; Kogut et al., 1992; Podolny, 1994). Caratteristiche simili favoriscono una maggiore "absorptive capacity" (Cohen e Levinthal, 1990), ovvero la capacità da parte delle organizzazioni di comprendere meglio il comportamento di potenziali partner, producendo come risultato una riduzione della probabilità di "azzardo morale", ossia di comportamenti opportunistici, e alimentando la convenienza ad instaurare relazioni di scambio. 
In una prospettiva diversa, Barney e Hoskisson (1990) si basano sull'idea dell'unicità delle risorse possedute da un'organizzazione - capacità e conoscenze del capitale umano, tecnologie e prodotti specifici - per spiegare il comportamento competitivo. Barney (1991), in particolare, sottolinea l'importanza per un' organizzazione di possedere caratteristiche distintive al fine di ottenere un vantaggio competitivo che sia sostenibile nel tempo. In questo senso, l'eterogeneità rispetto a determinate caratteristiche organizzative rappresenta un fattore che favorisce lo sviluppo di relazioni competitive all'interno di uno specifico settore. Tuttavia, proprio l'unicità di tali caratteristiche può spingere organizzazioni diverse a ricercare accordi e rapporti di collaborazione (Kock, 1991). Attivando comportamenti collaborativi, infatti, le organizzazioni accedono a risorse e competenze che sono tipiche di altre organizzazioni, sfruttando i vantaggi derivanti dalla condivisione e dalla eliminazione dei costi relativi allo sviluppo di risorse strategiche di valore. Soprattutto in settori ad alta intensità tecnologica la presenza di risorse eterogenee, complementari e asimmetriche è alla base di importanti accordi interorganizzativi e partnership di vario tipo (Teece, 1990; Shenhar, 2001).

\subsection{Ipotesi di ricerca}

Il quadro teorico appena descritto permette di evidenziare che il legame tra collaborazione e competizione sia tutt'altro che semplice da analizzare, sia sul piano teorico che empirico, e che caratteristiche organizzative simili o diverse possono allo stesso tempo spiegare comportamenti competitivi o piuttosto soluzioni tese alla collaborazione. Nel tentativo di riconciliare visioni teoriche diverse e apparentemente contrastanti, nel presente lavoro sviluppiamo un modello che considera l'effetto che l'interdipendenza competitiva esercita sulla propensione delle organizzazioni a collaborare.

L'interdipendenza competitiva è stata analizzata in letteratura utilizzando il concetto della niche overlap. Per niche overlap si intende il grado di sovrapposizione nel segmento o nei vari segmenti di nicchia da cui le imprese attingono risorse per la propria sopravvivenza (Hannan e Freeman, 1977, 1989). Il concetto di nicchia rappresenta, nell'ambito della teoria ecologica, l'elemento che permette di identificare una popolazione organizzativa, vale a dire un insieme di organizzazioni che dipendono da risorse simili e che condividono la stessa forma organizzativa (Freeman e Hannan, 1983). Una nicchia, in particolare, costituisce un particolare segmento di risorse o categoria di input/output produttivi che risultano fondamentali per la sopravvivenza di una popolazione organizzativa o di una singola organizzazione. Hannan e Freeman (1977: 947), in particolare, affermano che la nicchia "...consists of all those combinations of resource levels at which the population can survive and reproduce itself'. All'aumentare del numero di organizzazioni che dipendono dalle medesime risorse vi sarà un aumento nel livello di sovrapposizione delle organizzazioni nella nicchia e, di conseguenza, un incremento dell'intensità competitiva tra le stesse. 
Se da un lato una maggiore sovrapposizione nella nicchia di risorse implica dipendenza da risorse simili e maggiore competizione tra organizzazioni, dall'altro la compresenza in molteplici nicchie di risorse può generare comportamenti collaborativi. Questa argomentazione è in linea con il filone teorico della mutual forbearence, ovvero del "vivi e lascia vivere" (tra gli altri, Scott, 2001; Hughes e Oughton, 1993; Baum e Korn, 1996; Gimeno e Woo, 1996, 1999; Boeker et al., 1997). Secondo questa prospettiva, costi e benefici che le organizzazioni attribuiscono alla competizione sono influenzati in misura rilevante dall'ampiezza dello spazio competitivo. La consapevolezza reciproca di una maggiore interdipendenza competitiva da parte di organizzazioni che competono simultaneamente in molteplici mercati può determinare una revisione degli schemi competitivi, inducendo comportamenti collaborativi o quanto meno tesi al "vivi e lascia vivere". L'idea che la niche overlap possa indurre comportamenti di mutual forbearence è stata originariamente attribuita a Edwards (1955), secondo il quale "When one large conglomerate enterprise competes with another, the two are likely to encounter each other in a considerable number of markets. The multiplicity of their contact may blunt the edge of their competitor" (Edwards, 1955: 84). Poiché competere simultaneamente in più mercati permette all'attore organizzativo di avere accesso a informazioni importanti relativamente a strategie, modelli organizzativi e competenze di imprese rivali (Jayachandran et al., 1999), organizzazioni con elevati livelli di niche overlap troveranno più conveniente astenersi dal competere e ricercare soluzioni di collaborazione mutuamente vantaggiose (Barnett, 1993; Greve, 2006). I vantaggi associati all'informazione e alla conoscenza dell'ambiente competitivo rendono in sostanza possibile trasformare relazioni di natura competitiva in legami di scambio e di collaborazione tra organizzazioni. In questo senso, la competizione non è "antitetica" alla collaborazione; al contrario, essa può rappresentare uno strumento attraverso cui le imprese si avvicinano ai propri concorrenti. Ciò complessivamente permette di formulare la nostra prima ipotesi di ricerca:

Ipotesi 1 (HP1): L'interdipendenza competitiva tra coppie di organizzazioni alimenta la possibilità che le stesse instaurino legami di natura collaborativa.

L'ipotesi 1 assume una relazione monotonica positiva tra competizione e collaborazione. Tuttavia, organizzazioni che nell'ambito di una medesima popolazione acquisiscono risorse dalla stessa nicchia tenderanno ad assumere struttura e caratteristiche simili. Tale fenomeno, di omogeneità tra organizzazioni o isomorfismo, si spiega osservando che organizzazioni che occupano una stessa nicchia di risorse si trovano ad affrontare pressioni ambientali e condizioni competitive simili. Come conseguenza, esse manifesteranno similarità rilevanti in tutta una serie di importanti variabili organizzative (struttura, dimensione, attività, ecc.) (Hannan e Freeman, 1977). Alla luce di tali osservazioni, è possibile affermare che l'eccessiva sovrapposizione nell'ambito di una stessa nicchia può determinare, oltre un certo livello, un incremento dei costi associati alla mancanza di eterogeneità, riducendo allo stesso tempo i vantaggi 
informativi che la multipoint competition consente di realizzare. Tali costi riguardano, in particolare, la perdita di complementarietà nelle attività e nelle competenze rilevanti in un'ottica di potenziale collaborazione da parte di organizzazioni concorrenti (Aiken e Hage, 1968; Richardson, 1972). Il risultato che si osserva in presenza di una eccessiva sovrapposizione nella nicchia - $\mathrm{o}$, in altri termini, la dipendenza dalle medesime risorse - è dunque una riduzione delle possibilità di osservare scambi di risorse tra organizzazioni. Tenendo in considerazione la prima ipotesi di ricerca, sulla base di tali argomentazioni argomentiamo quanto segue:

Ipotesi 2 (HP2): Esiste una relazione a $U$ rovesciata tra il grado di interdipendenza competitiva e la possibilità di osservare relazioni di scambio tra coppie di organizzazioni.

\section{Disegno della ricerca}

\subsection{Il contesto istituzionale}

Il settore della sanità rappresenta un setting particolarmente ideale per condurre questa ricerca, in quanto caratterizzato da elementi che allo stesso tempo favoriscono la collaborazione ed alimentano la competizione a livello di sistema (Plochg et al., 2006). Pur mantenendo un elevato livello di autonomia gestionale ed organizzativa, le organizzazioni collaborano e integrano le proprie attività al fine di rispondere in modo efficace e tempestivo alle esigenze degli utenti/pazienti. In ambiti quali, ad esempio, la medicina di comunità e i servizi sociali, il soddisfacimento dei bisogni assistenziali del paziente richiede un elevato livello di coordinamento ed integrazione da parte di una molteplicità di organizzazioni che offrono servizi specialistici differenti (Lambrew et al., 1993; Provan e Milward, 1995). Alla luce delle diverse trasformazioni istituzionali che nel tempo si sono realizzate per superare le inefficienze, la scarsa qualità o le difficoltà equitative a livello di sistema, il dualismo competizione/cooperazione si è affermato come caratteristica qualificante dei principali sistemi sanitari internazionali.

Il focus della nostra ricerca è sul sistema sanitario della regione Lazio, parte integrante del più ampio Sistema sanitario nazionale $(\mathrm{Ssn})$. Il processo di devoluzione - dallo Stato alle Regioni - di importanti aspetti inerenti la regolamentazione, il finanziamento e le procedure di acquisto dei servizi, ha caratterizzato il panorama della sanità italiana negli ultimi decenni. Alla luce dell'attuale modello di sanità, alla singola Regione viene attribuita ampia autonomia per quanto riguarda la programmazione delle attività, l'allocazione delle risorse finanziarie e l'organizzazione dei servizi a livello territoriale.

Il Lazio conta una popolazione che si attesta intorno ai 5,3 milioni di abitanti, il 60\% dei quali concentrati nella Capitale. L'organizzazione del sistema è affidata a dodici Aziende Sanitarie Locali (ASL) che si avvalgono di un cer- 
to numero di strutture ospedaliere per la cura e il ricovero dei pazienti caratterizzate da forte eterogeneità nella natura giuridica e nelle caratteristiche strutturali e operative. Nel Lazio gli istituti di ricovero e cura sono complessivamente 164, classificati in quattro grandi tipologie: pubblici (aziende ospedaliere, presidi di ASL), equiparati al pubblico (policlinici universitari, istituti di ricovero e cura a carattere scientifico - cosiddetti Irccs -, e ospedali classificati), privati accreditati (case di cura accreditate) e privati non accreditati. Le prime tre categorie rappresentano l'ossatura dell'offerta assistenziale regionale, con un numero di posti letto che è complessivamente pari a circa 23.200 unità.

Nell'insieme tali strutture formano un modello istituzionale che Mapelli (2007) ha recentemente identificato come sistema "misto-quasi separato". In tale modello si assiste alla compresenza di elementi cooperativi e competitivi tra organizzazioni pubbliche e private operanti nell'ambito del sistema pubblico Regionale. Tali elementi sono in larga parte dovuti a determinanti normative che regolano il funzionamento generale del sistema sanitario regionale. Tra queste norme, quelle che favoriscono la competitività riguardano l'obbligo per le aziende sanitarie (sia aziende ospedaliere che territoriali, le ASL) di raggiungere l'equilibrio economico fra costi e ricavi d'esercizio e la valutazione del direttore generale sulla base del conseguimento di tale obiettivo, oltre che di obiettivi legati alla programmazione sanitaria regionale. Anche il meccanismo tariffario di remunerazione delle prestazioni ospedaliere rappresenta un fattore che alimenta la competizione tra provider di offerta. Grande rilevanza riveste infine nella sanità italiana la possibilità per il cittadino di esercitare la propria libertà di scelta nei confronti dei diversi fornitori di prestazioni, pubblici e privati. Anche se caratterizzato dalla presenza di elementi che alimentano la competizione tra provider di servizi, il sistema sanitario regionale preso a riferimento prevede un assetto istituzionale volto a favorire la collaborazione tra le organizzazioni e uno sviluppo armonico della rete di offerta dei servizi. Il primo elemento a sostegno della collaborazione è l'articolazione territoriale di questa rete; a livello regionale si assiste infatti ad una suddivisione fra ospedali focalizzati sull'erogazione di prestazioni ospedaliere di elevata complessità, e quelli che offrono servizi di medio-bassa complessità. Un secondo aspetto che incide sul livello di collaborazione interorganizzativa riguarda la determinazione dei volumi produttivi. Le Regioni hanno infatti adottato uno schema di finanziamento che prevede il trasferimento di fondi alle organizzazioni sanitarie sulla base di un budget globale definito attraverso una contrattazione tra singolo ospedale e autorità regionale, che permette l'assegnazione a ciascuna struttura (pubblica o privata) di un tetto di produzione e quindi un tetto complessivo di fatturato. Ciò limita di fatto la competizione tra ospedali per ciò che riguarda i volumi di pazienti da trattare. Un ultimo aspetto concerne infine il meccanismo dell'autorizzazione e dell'accreditamento, ovvero il processo istituzionale attraverso il quale le Regioni assegnano alle strutture sanitarie la facoltà di accedere ed operare per conto e a carico del Ssn. L'autorizzazione garantisce alle organizzazioni la possibilità di svolgere attività di assistenza sanitaria nell'ambito della Regione di appartenenza. L'accreditamento, 
che segue l'autorizzazione, inserisce invece la struttura sanitaria tra i "fornitori" di prestazioni del sistema pubblico e erogando prestazioni a carico del Ssn.

\subsection{Raccolta dei dati ${ }^{2}$}

In questo paper utilizziamo due tipologie di dati: di tipo relazionale, ovvero relativi all'interazione diadica tra coppie di provider di offerta presenti sul territorio regionale, e dati di tipo attributivo, riferiti sia agli asset che ai volumi di attività delle organizzazioni esaminate.

I due tipi di dati sono stati raccolti attraverso una survey ad hoc, condotta attraverso l'utilizzo di un questionario inviato alle strutture ospedaliere regionali, e attraverso il database del Sistema Informativo Ospedaliero (SIO), gestito dall'Agenzia di Sanità Pubblica della Regione Lazio. Il SIO, in particolare, rileva e gestisce informazioni analitiche su tutti i ricoveri ospedalieri (in acuzie e post-acuzie attraverso la SDO - Scheda di Dimissione Ospedaliera) che ogni anno si registrano negli istituti della Regione; produce annualmente rapporti sulle attività di ricovero e cura nella Regione e costituisce una fonte di dati per ricerche e studi epidemiologici e di valutazione e programmazione dei servizi; archivia inoltre informazioni sulla dotazione dei posti letto delle strutture di ricovero e gestisce la rilevazione giornaliera dell'occupazione dei letti per acuti. Un questionario è stato inoltre inviato nel corso del 2003 a tutti gli ospedali regionali ad eccezione dei privati non accreditati (operanti in solo regime privatistico). Il questionario, articolato in sezioni, ha permesso di raccogliere dati su: tipologia di struttura, risorse umane e professionali impiegate, sistemi informativi adottati, tipologia di servizi ospedalieri e tecnologie sanitarie.

Con un tasso di compliance pari a circa il 73\%, 91 questionari relativi ad altrettanti ospedali sono stati complessivamente ricevuti ed utilizzati nell'analisi. In ordine di numerosità, il $51 \%$ dei rispondenti è rappresentato da presidi ospedalieri delle ASL, il 29\% da strutture private accreditate, il 7\% da istituti classificati (ovvero ospedali dipendenti da istituti o enti ecclesiastici civilmente riconosciuti), il $10 \%$ da policlinici universitari e aziende ospedaliere e il rimanente 3\% da Istituti di ricerca e cura a carattere scientifico (Irccs). Queste strutture costituiscono complessivamente il campione della nostra indagine.

\subsection{Variabili attributive e relazionali}

Con la presente analisi intendiamo investigare l'effetto che l'interdipendenza competitiva (variabile indipendente) esercita sulla propensione delle organizzazioni a sviluppare rapporti di scambio e collaborazione (variabile dipen-

2. Parte dei dati utilizzati nel presente studio provengono da un più vasto progetto di ricerca denominato MINOREL. Gli autori ringraziano il gruppo di ricerca per aver messo a disposizione una parte dei dati raccolti nell'ambito di tale progetto. 
dente). Nell'esplorare il legame tra collaborazione e competizione, includiamo nell'analisi, come variabili di controllo, una serie di caratteristiche legate alle organizzazioni considerate, selezionate sulla base di una precisa scelta effettuata dagli autori in relazione alla rilevanza di tali fattori per la spiegazione dei fenomeni delle collaborazioni interorganizzative. Sono proprio queste ultime variabili che per prime andiamo a descrivere.

\subsubsection{Variabili attributive}

Le variabili attributive considerate nell'analisi si distinguono in variabili strutturali e di attività. Per quanto riguarda le prime, esse includono:

- Tipologia di struttura. Si considera la tipologia istituzionale dei diversi ospedali presi in esame attraverso una variabile categorica che distingue tra le sei principali identità istituzionali: aziende ospedaliere, presidi di ASL, policlinici universitari, Irccs (Istituti di ricovero e cura a carattere scientifico), ospedali classificati e case di cura accreditate.

- Livello assistenziale. Attraverso l'utilizzo di una variabile dummy si distingue il livello assistenziale offerto da tutte le strutture residenziali del territorio. In particolare, viene attribuito valore "1" alle organizzazioni ospedaliere a più alta specializzazione e i cui servizi possono essere classificati nell' ambito delle cure terziarie, mentre il valore " 0 " a tutte le altre strutture.

- Tecnologie sanitarie. Si utilizza una variabile binaria che fornisce indicazioni sul tipo di tecnologie - e corrispondenti servizi - utilizzate da un'organizzazione. Si tratta, più in particolare, delle seguenti tipologie: diagnostica di laboratorio, diagnostica per immagini e blocco operatorio. Le variabili corrispondenti assumono valore " 1 " nel caso di presenza, all'interno della struttura, di tutte e tre le tipologie di servizio, mentre assumono valore " 0 " in caso contrario.

- Numero di posti letto. È il numero di letti che una struttura ospedaliera allestisce e prepara per accogliere i pazienti, computati annualmente. Solitamente gli ospedali con un maggior numero di posti letto mettono a disposizione dell'utenza una più vasta gamma di servizi. Si tratta di una variabile continua che fornisce informazioni sulla dimensione di un'organizzazione.

- Numero dei dipendenti. È il numero totale di personale strutturato (medico, infermieristico, tecnico e amministrativo), rilevato attraverso il questionario e confrontato con il dato ufficiale del Ministero della Salute.

- Indice di managerialità. È un indicatore che considera il numero di personale amministrativo rispetto al numero totale di personale impegnato in attività assistenziali (medici, infermieri e tecnici). Tale indicatore fornisce informazioni sul grado di managerialità della struttura, ovvero sulla presenza, all'interno della stessa, di un orientamento verso l'utilizzo di modelli e strumenti di gestione orientati all'efficienza e alla flessibilità. 
- Grado di informatizzazione. È un indicatore volto a rilevare la presenza, nella struttura, di strumenti informatici e sistemi informativi aziendali. In particolare, attraverso il questionario è stato chiesto di indicare la presenza nell'organizzazione di strutture di coordinamento dei sistemi informativi aziendali, di programmi di registrazione dei dati sulle dimissioni ospedaliere e di archivi elettronici di registrazione in linea dei posti letto disponibili, delle sale operatorie impegnate, ecc.

- Distanza geografica. Si considera, inoltre, la distanza geografica media, misurata in chilometri, tra le diverse strutture residenti nel territorio regionale. Tale variabile fornisce un'idea della centralità di una struttura all'interno della rete di offerta dei servizi o, in altri termini, della distanza media di una struttura rispetto a tutte le altre presenti sul territorio regionale.

- ASL di appartenenza. Si tiene conto infine, nel modello, di una variabile categorica che controlla per l'appartenenza delle diverse strutture alle ASL di riferimento. L'appartenenza alla medesima azienda territoriale incide infatti sul livello di cooperazione sviluppato tra le strutture.

Oltre alle suddette informazioni su alcune caratteristiche strutturali delle organizzazioni investigate, $i$ dati raccolti si riferiscono anche alla tipologia e ai volumi di attività. Questi dati si riferiscono, nello specifico, al numero dei pazienti dimessi, all'indice di case-mix, alla degenza media e totale, alla percentuale di DRG chirurgici e ai ricavi totali.

- Numero di pazienti dimessi. Ė il numero totale dei pazienti dimessi dalle diverse strutture ospedaliere regionali nell'arco di un anno e fornisce indicazioni sulla dimensione di un'organizzazione.

- Indice di Case Mix (ICM). È un indice di attività che mette a confronto la complessità della casistica trattata da una struttura rispetto alla casistica di una struttura di riferimento, in genere considerata come "universo" (ad esempio, la complessità dei casi dell'ospedale A rispetto a tutti gli altri ospedali della Regione).

- Giornate di Degenza. Corrisponde al numero di giornate di ricovero registrate in un anno per tutti i pazienti trattati.

- Degenza media. E espressa dal rapporto tra il numero totale di giornate di ricovero e il numero totale di dimissioni ospedaliere. Sia la degenza totale che la degenza media possono influenzare l'offerta di servizi sanitari in modo diverso. La degenza media generalmente riflette la maggiore complessità legata alla casistica dei pazienti, ma può anche essere considerata un indicatore di efficienza nello svolgimento delle attività ospedaliere.

- DRG chirurgico. Tale indicatore fornisce informazioni sul tipo di struttura e sulle connesse attività assistenziali. Il DRG chirurgico, in particolare, indica la presenza di un intervento chirurgico o di altra procedura "indicativa" (intesa come tale dal sistema di classificazione DRG) in relazione ad una specifica diagnosi principale.

- Ricavi totali. È una variabile che esprime il fatturato complessivo annuo conseguito dalle strutture ospedaliere, sia in regime di ricovero ordinario che in Day Hospital. 
Per riassumere, la Tabella 1 contiene l'elenco delle variabili attributive considerate nell'analisi, insieme ai valori minimo e massimo, alla media e alla standard deviation associati a ciascuna di esse.

Tab. 1 - Statistiche descrittive delle variabili attributive $(n=91)$

\begin{tabular}{lcrrrr}
\hline Variabile & Tipologia & Min & Max & Media & Dev. Standard \\
\hline Degenza Media & Continua & 2,7 & 15,2 & 7,116 & 2,040 \\
Degenza Totale & Continua & 566 & 554.932 & $60.591,96$ & $92.383,15$ \\
Case Mix & Continua & 0,680 & 1,282 & 0,945 & 0,108 \\
DRG Chirurgici (\%) & Continua & 0 & 92 & 32,604 & 20,389 \\
N Posti Letto & Continua & 22 & 1968 & 230 & 307 \\
Blocco Operatorio & Binaria & 0 & 1 & - & - \\
Diagnostica Immagini & Binaria & 0 & 1 & - & - \\
Diagnostica Labor. & Binaria & 0 & 1 & - & - \\
Informatizzazione & Continua & 0 & 2,83 & 1,86 & 0,720 \\
Managerialità struttura & Continua & 0 & 1,71 & 0,14 & 0,235 \\
N Dipendenti & Continua & 11 & 2.924 & 398 & 484 \\
Tipologia Istituzionale & Categorica & 1 & 6 & - & - \\
Livello assistenziale & Binaria & 0 & 1 & - & - \\
ASL di appartenenza & Binaria & 0 & 1 & - & - \\
N Pazienti Dimessi & Continua & 101 & 94.044 & 11.983 & 17.145 \\
Distanza geografica (Km) & Continua & 29,6 & 120,0 & 48,6 & 24,5 \\
Ricavi (Euro) & Continua & 250.334 & 245.092 .200 & 26.589 .858 & 39.755 .779 \\
\hline & & & & -
\end{tabular}

\subsubsection{Variabili relazionali}

I dati relazionali si riferiscono ai rapporti di collaborazione e di competizione tra gli ospedali che compongono il campione. Entrambe le variabili, dipendente e indipendente, sono dunque variabili relazionali. Come anticipato, $i$ dati per la costruzione di queste variabili sono stati ottenuti attraverso il database SIO, anno 2003. La Tabella 2 contiene le statistiche descrittive relative alle due matrici di collaborazione e competizione.

Per quanto riguarda la prima tipologia di relazioni, il trasferimento interorganizzativo di pazienti rappresenta una delle modalità principali attraverso le quali è possibile osservare rapporti di scambio e collaborazione tra organizzazioni (Levin e White, 1961). I dati di cui disponiamo si riferiscono ai flussi di risorse tra ospedali - rilevati a livello di diade - misurati in termini di numero di pazienti trasferiti tra le varie strutture, pubbliche e private accreditate, presenti sul territorio regionale ${ }^{3}$. Sulla base di tali dati è stato possibile ricostruire

3. Lo scambio a cui ci riferiamo nello studio contempla il caso di un paziente ammesso nell'ospedale $i$ che viene da quest'ultimo inviato all'ospedale $j$. Normalmente, tale trasferimento avviene in caso di carenza di posti letto nell'ospedale di ricezione del paziente, man- 
Tab. 2 - Statistiche descrittive delle variabili relazionali

\begin{tabular}{lrr}
\hline & $\begin{array}{c}\text { Flusso trasferimento } \\
\text { pazienti* }\end{array}$ & $\begin{array}{c}\text { Competizione } \\
\text { (Niche Overlap) }\end{array}$ \\
\hline Media (Densità) & 0,098 & 0,088 \\
Dev Standard & 0,297 & 0,162 \\
Min & 0 & 0,000004 \\
Max & 1 & 0,983 \\
N Osservazioni (nodi) & $8190(91)$ & $8190(91)$ \\
OutDegree medio (Dev Stand) & $9,710(11,212)$ & $7,902(6,792)$ \\
InDegree medio (Dev Stand) & $9,710(10,454)$ & $7,902(6,430)$ \\
Centralizzazione (OutDegree) & $49,270 \%$ & $46,083 \%$ \\
Centralizzazione (InDegree) & $38,047 \%$ & $33,479 \%$ \\
OutDegree Max (Min) & $58(0)$ & $48,241(0,515)$ \\
InDegree Max (Min) & $47(0)$ & $37,208(0,314)$
\end{tabular}

* Matrice dicotomizzata rispetto al valor medio della matrice $\mathrm{X}$ per cui $\mathrm{x}_{\mathrm{ij}}=1$ se $\mathrm{x}_{\mathrm{ij}}>$ media, $x^{\prime}{ }_{i j}=0$ in caso contrario

una matrice sociometrica di dimensione $91 \times 91$. Su ciascuna riga e colonna della matrice sono riportate le diverse strutture ospedaliere che, rispettivamente, inviano e ricevono pazienti, mentre le celle di intersezione contengono il numero di pazienti che transitano da una struttura all'altra. La matrice è asimmetrica, poiché il numero di pazienti trasferiti non necessariamente coincide con il numero di pazienti ricevuti.

La seconda tipologia di legami riguarda le relazioni di natura competitiva che si stabiliscono tra coppie di ospedali. Misurare la competizione tra ospedali concettualizzandola come una relazione è piuttosto innovativo. Vari studi in passato hanno misurato l'interdipendenza competitiva tra organizzazioni osservando in che misura, nell' ambito di una comunità organizzativa, si verificano livelli di overlap rispetto a certe nicchie di risorse (Hannan e Freeman, 1989; Burt, 1992; Burt e Talmud, 1993).

Utilizzando la metodologia proposta da Sohn $(2001,2002)$ l'interdipendenza competitiva tra due organizzazioni $i$ e $j$ viene calcolata attraverso un coefficiente di competizione, $\mathrm{C}_{\mathrm{ij}}$, che misura, per ogni organizzazione, l'intensità della competizione che $i$ riceve da $j$ e viceversa. Tale misura consente altresì di verificare il grado di asimmetria nelle relazioni competitive tra coppie di organizzazioni. Ai fini del calcolo del coefficiente, il punto di partenza è rappresentato da una matrice, di dimensioni " $\mathrm{x}$ m", che esprime la relazione tra singole organizzazioni e nicchie di risorse. In particolare, sulle righe della matrice si riportano le organizzazioni considerate nel campione, mentre sulle colonne i vari segmenti da cui tali organizzazioni attingono le risorse necessarie per la sopravvivenza, cioè nel nostro caso i pazienti. I segmenti possono esse-

canza di competenze per la risoluzione del problema di salute del paziente, oppure un livello di assistenza richiesto dalla patologia diverso o superiore rispetto a quello fornito dall'ospedale che trasferisce il paziente stesso. 
re rappresentati, ad esempio, dalla zona in cui risiedono i pazienti o dalle rispettive aree sanitarie di pertinenza assegnate nell'ambito di sistemi sanitari in cui è presente una programmazione dei servizi a livello regionale. Il totale dei diversi vettori riga esprime dunque l'ammontare complessivo di risorse, cioè $i$ pazienti, che la singola organizzazione acquisisce dai vari segmenti di mercato (aree zonali o sanitarie). Nel caso specifico, la matrice " $\mathrm{x}$ m" contiene nelle righe le strutture ospedaliere della Regione e nelle colonne le diverse ASL in cui è suddivisa territorialmente la regione Lazio. Le ASL, come è noto, rappresentano delle articolazioni territoriali ben delineate del sistema sanitario regionale, nelle quali la tipologia e le modalità di erogazione dei servizi sono disegnate in base alle specifiche esigenze assistenziali della popolazione residente e in base alle caratteristiche infrastrutturali e tecnologiche delle organizzazioni che si distribuiscono sul territorio. Ogni cella di intersezione della matrice contiene il numero di pazienti residenti in una data ASL curati presso ciascuno degli ospedali della Regione. Poiché il Lazio è articolato in dodici ASL, la matrice di incidenza ricostruita in questa prima fase è di dimensione "91 x 12". I coefficienti di competizione sono stati calcolati con la seguente formula (Sohn, 2001):

$$
C_{i j}=\frac{\sum_{k} x_{i k} \min \left(x_{i k}, x_{j k}\right)}{\sum_{k} x_{i k}^{2}}
$$

dove $C_{i j}$ è l'indice di intensità competitiva che misura il livello di overlap nella nicchia di risorse tra gli ospedali $i$ e $j$; il numeratore è dato dalla sommatoria dei livelli di sovrapposizione tra coppie di ospedali nei diversi segmenti, mentre il denominatore esprime l'ampiezza complessiva della nicchia per ciascuna organizzazione, ovvero il numero complessivo dei pazienti ammessi. Il fattore $\min \left(\mathrm{x}_{\mathrm{ik}}, \mathrm{x}_{\mathrm{jk}}\right)$ implica che il coefficiente $\mathrm{C}_{\mathrm{ij}}$ sia compreso tra 0 (assenza di overlap) e 1 (overlap completo). Attraverso il computo dei coefficienti di competizione per ciascun ospedale, la matrice di partenza è stata trasformata (con l'ausilio del software MATLAB) in una matrice quadrata one-mode "91 x 91", le cui celle di intersezione esprimono l'intensità competitiva tra coppie di ospedali. Al fine di testare la nostra seconda ipotesi di ricerca è stata infine elaborata una seconda matrice di competizione, $\mathrm{i}$ cui valori di cella sono stati ottenuti elevando al quadrato i valori di cella della matrice originaria.

\section{Modello statistico}

Le ipotesi formulate sono state testate attraverso un'analisi di regressione multivariata per dati relazionali, anche nota come Multiple Regression Quadratic Assignment Procedure (Hubert, 1987; Krackhardt, 1988; Butts e Carley, 
2001) ${ }^{4}$. La MRQAP è un algoritmo statistico non parametrico che realizza la regressione della matrice dipendente in funzione di una o più matrici indipendenti. L'algoritmo procede in due fasi distinte. Nella prima viene realizzata una regressione che confronta le celle della matrice dipendente con le celle corrispondenti delle matrici indipendenti. Successivamente, le righe e le colonne della matrice dipendente vengono permutate casualmente e il modello di regressione viene ricalcolato. L'algoritmo ripete il processo di permutazione più volte (nel nostro caso 2000) per valutare la significatività del modello di regressione.

Nel nostro caso, la matrice che rappresenta la variabile dipendente è costituita dai rapporti di collaborazione tra ospedali. Dato un network rappresentato da una generica matrice $Y$ di tipo quadrato "n x n", il valore $y_{i j}$ è uguale a zero se gli attori $i$ e $j$ non sono legati da una relazione di scambio, mentre è uguale ad un valore intero positivo in caso contrario. La rappresentazione strutturale di una generica variabile relazionale può essere formalizzata come segue:

$$
Y=\left(\begin{array}{cccc}
0 & y_{1,2} & \ldots & y_{1, n} \\
y_{2,1} & 0 & \ldots & y_{2, n} \\
\ldots & \ldots & 0 & \ldots \\
y_{n, 1} & y_{n, 2} & \ldots & 0
\end{array}\right)
$$

Il modello econometrico di riferimento per l'analisi di correlazione condotta nel presente studio è il seguente:

$$
y_{i j}^{c o o p}=\alpha+\beta x_{i j}+\varepsilon_{i j}
$$

dove $y_{i j}$ rappresenta la variabile (matrice) dipendente del modello, che esprime la presenza di relazioni di cooperazione tra le organizzazioni $i$ e $j$, mentre la matrice $x_{i j}$ contiene l'insieme delle variabili esplicative relative a $i$ e $j$. Tra queste variabili identifichiamo l'intensità competitiva tra coppie di attori, misurata attraverso la misura di niche overlap precedentemente illustrata, ed altre variabili di controllo, su cui non sviluppiamo teoria in questo paper ma che potenzialmente spiegano la varianza della variabile dipendente considerata. Queste variabili, precedentemente illustrate, riguardano la dotazione di risorse degli ospedali (numero di posti letto e numero di dipendenti), i volumi di attività

4. L'analisi di correlazione non è stata effettuata utilizzando modelli OLS standard poiché la presenza di una auto-correlazione strutturale tra i valori di riga e quelli di colonna di una generica matrice mette in discussione le ipotesi di indipendenza delle osservazioni dei modelli di regressione standard e rende difficile interpretare i livelli di significatività dei coefficienti di regressione forniti normalmente attraverso i più comuni test statistici (Krackhardt, 1987). Un metodo alternativo per valutare la significatività dei coefficienti in casi di dati relazionali diadici prevede l'impiego di test QAP (quadratic assignment procedure). 
(numero di pazienti dimessi), la tipologia e la complessità delle attività assistenziali svolte (degenza media e totale, \% di DRG chirurgici, Case-mix, tipologia istituzionale), la complessità tecnologica (blocco operatorio, diagnostica per immagini, diagnostica di laboratorio), la distanza geografica, i ricavi complessivi. Come anticipato, il modello include inoltre una variabile dummy per tener conto del livello assistenziale garantito dalle organizzazioni sottoposte ad indagine (cure terziarie $v s$ altri livelli assistenziali) e una variabile categorica che considera le ASL di appartenenza degli ospedali ${ }^{5}$.

\section{Analisi e risultati}

L'analisi di regressione multivariata è stata condotta attraverso la tecnica del semi-partialling approach, utilizzando l'ormai diffuso software UCINET vers. 6.177 (Borgatti et al., 2002). L'obiettivo è quello di verificare quanto le diverse variabili indipendenti considerate (interdipendenza competitiva, caratteristiche strutturali e di attività) contribuiscono a spiegare lo scambio di pazienti tra strutture ospedaliere. I risultati ottenuti da quest'analisi sono illustrati in Tabella 3.

Come si osserva in Tabella, l'analisi è di tipo step-wise, con l'elaborazione di tre diversi modelli di regressione. I tre diversi modelli di regressione che presentiamo illustrano i diversi passi logici della nostra spiegazione. I risultati del Modello I evidenziano che sono complessivamente dieci le covariate nel modello significativamente associate al livello di cooperazione tra strutture ospedaliere, ossia la degenza media e totale dei ricoveri, la percentuale dei DRG chirurgici trattata, la numerosità dei dipendenti, il numero di pazienti dimessi, i ricavi, il livello assistenziale e la tipologia istituzionale afferenti al sin-

5. Al fine di analizzare in maniera diadica anche le variabili attributive relative alle organizzazioni, queste sono state trasformate in indicatori di differenza rispetto allo specifico attributo considerato. Più specificatamente, l'impiego dei test QAP richiede l'appropriata trasformazione di vettori di valori in matrici di adiacenza " $\mathrm{n}$ x $\mathrm{n}$ ". Partendo dai vettori di attributi relativi ad ogni singola struttura ospedaliera, è possibile ottenere delle matrici (anche indicate con il termine pseudo-network) i cui valori di cella non rispecchiano propriamente delle relazioni tra coppie di nodi, quanto piuttosto le differenze (ottenute utilizzando operazioni algebriche che variano al variare della variabile considerata) nei valori relativi alle variabili attributive considerate. Nel nostro caso, escludendo l'intensità competitiva già disponibile in forma matriciale, per la trasformazione delle variabili esplicative abbiamo utilizzato i seguenti criteri: per variabili categoriche e binarie la generica matrice $\mathrm{X}$ è data dal generico valore di cella $\mathrm{x}_{\mathrm{ij}}=1$ se vettore $(i)=$ vettore $(j)$ o da $\mathrm{x}_{\mathrm{ij}}=0$ in caso contrario (criterio exact matches); per le variabili continue la matrice $\mathrm{X}$ è invece il risultato di $\mathrm{x}_{\mathrm{ij}}=\mid$ vettore $(i)$ - vettore $(j) \mid$ (criterio absolute differences). L'unica eccezione riguarda la variabile relativa al livello di assistenza erogata, per la quale la trasformazione del relativo vettore di valori in matrice è stata eseguita seguendo un criterio diverso (quello del "prodotto"), al fine di distinguere tra i due gruppi di aziende ospedaliere, policlinici istituzionali e Irccs, da una parte, e case di cura accreditate o presidi ospedalieri di ASL, dall'altra, cui corrispondono livelli di assistenza erogata diversi. 


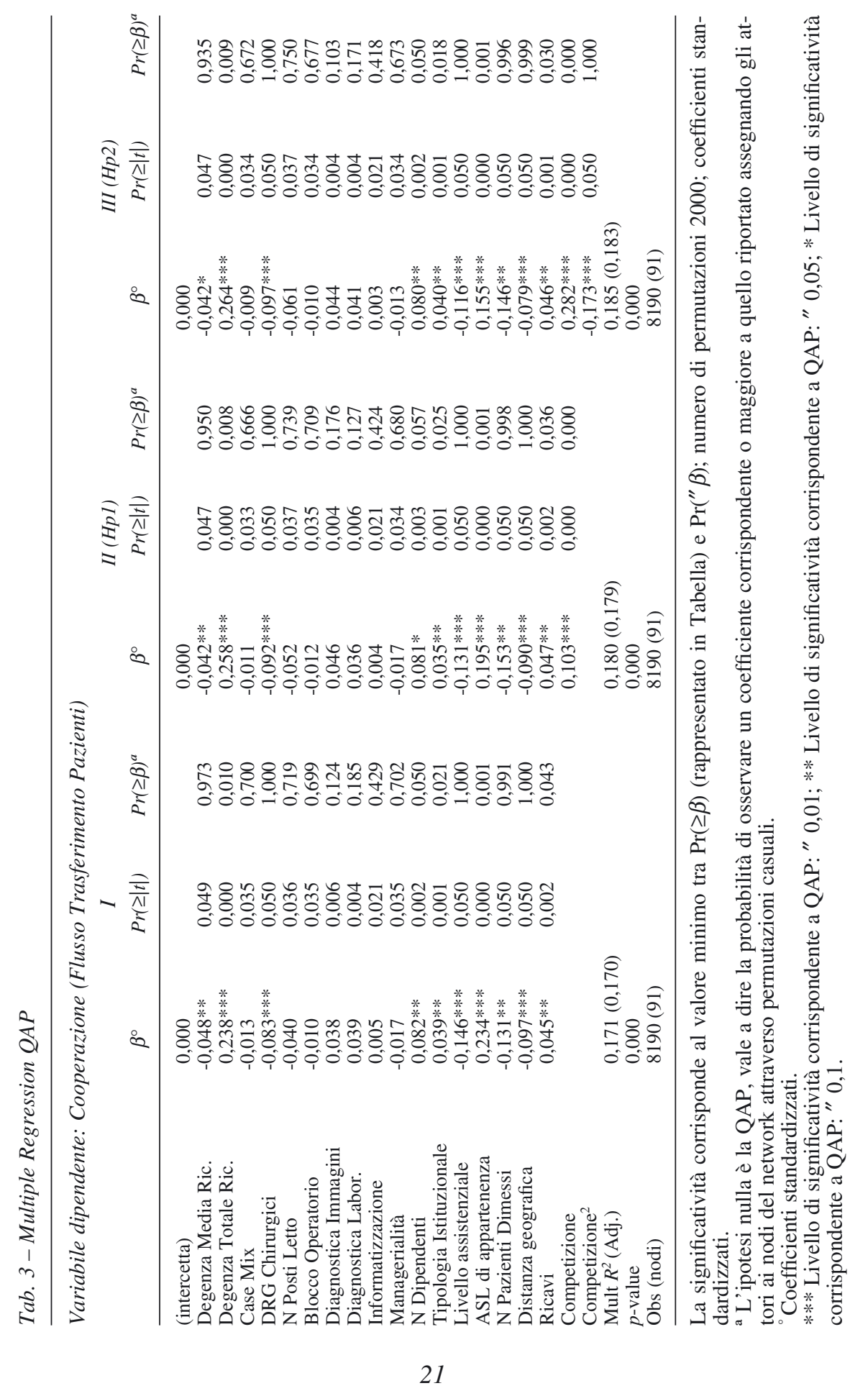


golo ospedale, l'ASL di appartenenza, la distanza geografica tra ospedali. Per quanto riguarda la variabile relativa alla degenza totale, poiché il coefficiente è positivo e significativo $\left(\beta=0,238 ; t^{\prime \prime} 0,01\right)$, e ricordando che il valore di cella è stato ottenuto con la differenza in valore assoluto tra $i$ vettori di valori relativi a coppie di organizzazioni, è possibile asserire che differenze nelle giornate di degenza prodotte erogate influenzano positivamente la probabilità di osservare relazioni di scambio tra ospedali. In altre parole, tanto più due organizzazioni sono eterogenee in termini di volumi di attività, tanto più sarà possibile osservare relazioni di scambio tra le stesse. Sembra plausibile immaginare legami collaborativi tra strutture piccole e grandi nell'ambito dei processi di cura dei pazienti. Come osservato in un recente studio, "clinical networks among very small and large hospitals is a way to create patterns for concentrating complex cases requiring a certain critical mass" (Lega e De Pietro, 2005: 265). Questo risultato sembra inoltre confermato da altre variabili che appaiono significativamente correlate all'intensità cooperativa: il numero di dipendenti e i ricavi. La variabile relativa al numero dei dipendenti presenta un coefficiente positivo e significativo $\left(\beta=0,082 ; t^{\prime \prime} 0,05\right)$ così come la variabile relativa ai ricavi $\left(\beta=0,045 ; t^{\prime \prime} 0,05\right)$, segnalando che la maggiore eterogeneità in termini di dimensione organizzativa aumenta la probabilità di osservare relazioni di scambio. In controtendenza appare invece il risultato relativo alla variabile numero di pazienti dimessi, anch'essa generalmente considerata una variabile che riflette la dimensione ospedaliera rispetto ai volumi di attività generati. In questo caso il coefficiente è negativo $\left(\beta=-0,131 ; t^{\prime \prime} 0,05\right)$, segnalando che strutture sanitarie simili rispetto al volume di pazienti trattati sono caratterizzati per una maggiore propensione a collaborare. Negativa è anche l'associazione rilevata tra la propensione degli ospedali a collaborare e le variabili che considerano degenza media dei pazienti $\left(\beta=-0,048 ; t^{\prime \prime} 0,05\right)$ e percentuale dei DRG chirurgici sul totale $\left(\beta=-0,083 ; t^{\prime \prime} 0,01\right)$. Questi risultati evidenziano che la similarità e l'omogeneità nelle attività svolte, soprattutto con riferimento ai livelli di efficienza nella gestione dei posti letto e nei volumi legati alla casistica dei pazienti chirurgici, influenza positivamente la generazione di relazioni di scambio tra ospedali. L'altra variabile che appare significativamente correlata alla variabile dipendente è la distanza geografica, che riporta un coefficiente negativo ( $\left.\beta=-0,097 ; t^{\prime \prime} 0,01\right)$, a testimonianza del fatto che la prossimità geografica tra due organizzazioni aumenta la possibilità di osservare legami cooperativi. Anche questo risultato è plausibile, poiché conferma che la distanza geografica rappresenta un ostacolo ai vantaggi che possono derivare dalla creazione di legami collaborativi. La relazione negativa con la distanza geografica sembra dunque confermare l'incentivo per organizzazioni che sono più vicine a creare legami di collaborazione. Interessante è, infine, il risultato che si riferisce alle variabili istituzionali. Il livello assistenziale offerto appare negativamente e significativamente correlato con la variabile dipendente $\left(\beta=-0,146 ; t^{\prime \prime} 0,01\right)$ evidenziando la tendenza da parte delle organizzazioni a collaborare in relazione al diverso livello assistenziale offerto 
(per esempio, assistenza di secondo livello $v s$ assistenza di alta specialità). I risultati mostrano inoltre che la variabile che riflette l'appartenenza alle varie categorie istituzionali appare positivamente correlata alla propensione degli ospedali a scambiare risorse $\left(\beta=0,039 ; t^{\prime \prime} 0,05\right)$ : strutture che afferiscono alla stessa categoria istituzionale sono più propense a scambiare risorse con maggiore intensità. Un'ultima variabile di tipo istituzionale è quella relativa all'ASL di appartenenza degli ospedali. Come si osserva in Tabella 3, il coefficiente in questo caso è positivo e significativo $\left(\beta=0,234 ; t^{\prime \prime} 0,01\right)$ segnalando così che l'appartenenza degli ospedali ad una stessa ASL influisce positivamente sulla propensione a scambiare pazienti.

Il modello II include, oltre alle variabili di controllo, anche la variabile che esprime l'interdipendenza competitiva tra organizzazioni. Come si osserva nella Tabella 3, tale variabile risulta positivamente e significativamente correlata con la propensione degli ospedali a collaborare $(\beta=0,103 ; t$ " 0,01$)$. I risultati evidenziano inoltre un aumento nel valore di goodness-of-fit del modello II rispetto al modello I $\left(R^{2}=0,180\right.$, contro $\left.R^{2}=0,171\right)$. Ciò complessivamente permette di confermare la nostra prima ipotesi di ricerca, ovvero che l'interdipendenza competitiva tra coppie di organizzazioni ospedaliere favorisce lo scambio di pazienti. Rispetto al modello I si osserva, inoltre, che i risultati della regressione non presentano variazioni significative per quanto riguarda le altre variabili indipendenti. Il segno dei coefficienti e la significatività delle variabili rimangono infatti costanti.

Il modello III considera infine anche la squared competition, ossia la misura di niche overlap elevata al quadrato. Tale modello consente di verificare la nostra seconda ipotesi di ricerca, la quale assume che per livelli di pressione competitiva elevati la propensione alla collaborazione diminuisce, controllando per tutte le altre variabili considerate. I risultati confermano l'ipotesi da noi formulata: il coefficiente per la variabile "competizione" rimane positivamente e significativamente correlato alla variabile dipendente $(\beta=0,282 ; t$ " 0,01$)$, mentre il parametro per la squared competition evidenzia una relazione negativa e significativa alle relazioni di scambio di pazienti tra ospedali $(\beta=-0,173$; $t^{\prime \prime}$ 0,01). Il valore della goodness-of-fit inoltre aumenta rispetto ai modelli II e I $\left(R^{2}=0,185\right)^{6}$.

6. I risultati dell'analisi sono stati corroborati effettuando alcune modifiche al modello di regressione. In primo luogo, è stata effettuata una trasformazione logaritmica di alcune delle variabili (ricavi, numero dei posti letto, giornate di degenza) al fine di evitare fenomeni di confondimento legati alla loro particolare distribuzione skewed, non rispondente quindi ad un andamento normale. È stata inoltre effettuata un'analisi di multicollinearità condotta sulle covariate incluse nel modello attraverso i Variance Inflation Rates (VIF), e si è riscontrato un VIF $>10$ proprio per le tre variabili sopra citate. Computando nuovamente il modello, sia con le variabili opportunamente trasformate, sia escludendo le covariate potenzialmente in grado di alterare coefficienti ed errori standard, non abbiamo riscontrato cambiamenti significativi nei risultati conseguiti. I risultati di queste analisi possono essere fornite dagli Autori su richiesta. 


\section{Discussione e Conclusioni}

Il presente lavoro si pone l'obiettivo di investigare il tema della collaborazione e della competizione tra organizzazioni, analizzando empiricamente l'effetto che la competizione esercita sulla propensione delle organizzazioni a collaborare.

Il filone teorico della population ecology utilizza il concetto di nicchia per individuare quelle organizzazioni, o popolazioni organizzative, che dipendono dalle medesime risorse per la propria sopravvivenza. Tanto più due organizzazioni dipendono da risorse simili, tanto più si troveranno a competere per l'acquisizione o il mantenimento di tali risorse. In tale prospettiva, la sovrapposizione nei segmenti di nicchia, o niche overlap, è considerata una misura di intensità competitiva che riduce e ostacola le opportunità di collaborazione $\mathrm{e}$ scambio (Dobrev e Kim, 2006; Dobrev, Kim e Hannan, 2001). In un'ottica diversa, tuttavia, la niche overlap - intesa nel senso di similarità nella dipendenza dalle medesime risorse - può anche incoraggiare la collaborazione. In effetti, organizzazioni con interessi e caratteristiche simili possono trovare convenienza a comunicare e coordinare le loro attività e dunque ad avviare una qualche forma di collaborazione. In generale, le principali forme di partnership interorganizzative trovano stabilità e hanno successo tanto più le strutture organizzative coinvolte sono simili in termini di attributi e di obiettivi (tra gli altri, Popielarz e McPherson, 1995). La coesistenza, dunque, di queste visioni alternative sul rapporto e l'influenza reciproca tra collaborazione e competizione consente di affermare che tale relazione non è lineare e che, soprattutto, è possibile considerare la collaborazione e la competizione come logiche diverse di un medesimo fenomeno, quello delle relazioni orizzontali tra imprese.

Nell'esplorare tali fenomeni, quello della sanità rappresenta senza dubbio un laboratorio ideale. Da un lato, infatti, appare chiaro - oltre che congruo con gli obiettivi di equità che il sistema sanitario si pone - che le organizzazioni sanitarie non possano esimersi dal collaborare, e i più recenti sviluppi del sistema di offerta dei servizi nel panorama sanitario nazionale e internazionale sembrano mostrare un orientamento sempre più marcato in questa direzione. Se è vero, però, che i sistemi sanitari sono improntati all'integrazione dell'azione organizzativa, è vero altresì che le riforme degli ultimi anni hanno delineato sistemi di programmazione delle attività, di finanziamento e di accreditamento delle strutture che introducono e alimentano meccanismi di competizione tra i diversi provider di offerta, pubblici e privati. È dunque possibile che due organizzazioni che competono per la sopravvivenza o per la tutela della quota di mercato siano allo stesso tempo chiamate a collaborare in nome di un preciso disegno istituzionale? Quali fattori spingono le organizzazioni a sviluppare rapporti di scambio con altre organizzazioni? Fino a che punto collaborazione e competizione possono coesistere? Queste sono le domande a cui il presente lavoro tenta di fornire una risposta, analizzando la situazione di un sistema sanitario, quello della regione Lazio, particolarmente interessante da un punto di vista di modello istituzionale adottato, contraddistinto dalla presenza 
di numerose strutture fortemente eterogenee nella natura giuridica e nelle caratteristiche strutturali e operative. I risultati ottenuti permettono di tracciare alcune considerazioni e di fornire indicazioni di natura teorica, metodologica e manageriale.

Una prima implicazione si collega al dibattito sulle dinamiche di collaborazione e di competizione interorganizzativa. L'analisi consente di osservare come le organizzazioni agiscono nel proprio ambiente instaurando legami multidimensionali e che la presenza di una interdipendenza competitiva può favorire, date certe caratteristiche organizzative, l'instaurarsi di relazioni di scambio tra organizzazioni. Tanto più due organizzazioni condividono e dipendono dalla medesima nicchia di risorse, tanto più avranno informazioni e conoscenze riguardo a caratteristiche reciproche che possono nel tempo incoraggiare legami di natura collaborativa. La competizione non implica necessariamente un conflitto tra organizzazioni, quanto piuttosto una rivalità tra strutture che - sotto certe condizioni - si trasforma in scambi di risorse che testimoniano comportamenti tesi alla collaborazione. Esiste, tuttavia, un livello di competizione oltre il quale la collaborazione non trova più spazio a fronte di un conflitto vero e proprio che viene a determinarsi tra strutture organizzative a causa di una eccessiva omogeneità, o mancanza di complementarietà. Specialmente in specifici comparti della sanità quali, ad esempio, la medicina di comunità, la cura delle malattie croniche, le tossicodipendenze e l'alcolismo, in cui organizzazioni diverse necessitano di integrare gli sforzi al fine di rispondere efficacemente alle esigenze degli utenti (Provan e Milward, 1995), si deve tenere conto degli effetti perversi che pressioni competitive accentuate potrebbero generare in termini di efficacia ed efficienza degli interventi. Se incentivare le strutture all'efficienza gestionale e alla razionalizzazione delle risorse favorendo la competizione tra organizzazioni non è dunque di per sé un elemento che compromette il livello di integrazione, i comportamenti competitivi tra ospedali dovrebbero essere attentamente monitorati al fine di evitare situazioni che mettano a rischio il livello di integrazione dei servizi stessi. In questa direzione appare utile tanto l'azione di vigilanza di soggetti istituzionali pubblici (ad esempio, le Regioni), quanto l'intervento di altri attori (le agenzie di antitrust) con il compito di controllare l'eccessiva competizione in settori in cui appare invece indispensabile un certo livello di collaborazione interorganizzativa.

Un altro importante aspetto nello studio dei fenomeni collaborativi riguarda l'omofilia, o di contro l'eterogeneità, come fattore decisivo per la formazione di relazioni di scambio. Alcuni studi hanno osservato che la similitudine tra organizzazioni in termini strutturali (considerando ad esempio la dimensione, le risorse finanziarie e la tipologia di struttura) di fatto facilita i rapporti di scambio tra imprese (Podolny, 1994; Barley et al., 1992). La similarità favorisce una maggiore capacità di assorbimento da parte delle organizzazioni rispetto ad opportunità di conoscenza reciproca, le quali generano a loro volta il presupposto per instaurare rapporti di collaborazione convenienti. Il nostro studio conferma solo in parte l'ipotesi di omofilia. I risultati evidenziano infat- 
ti, da un lato, che gli ospedali preferiscono collaborare con organizzazioni che svolgono attività similari per quanto riguarda l'efficienza nella cura dei pazienti (degenza media) e la tipologia di servizi offerti (\% DRG chirurgici), piuttosto che rivolgersi a strutture dissimili; dall'altro, tuttavia, emerge che la similitudine in termini di dimensione (misurata dal numero dei dipendenti, dal fatturato e dalle giornate di degenza complessive) e di livelli assistenziali offerti non necessariamente incide sulla propensione delle organizzazioni a collaborare. Alla luce di tali risultati non appare dunque possibile confermare in maniera univoca l'ipotesi di omofilia, o viceversa di complementarietà, quali fattori in grado di determinare la propensione da parte delle organizzazioni a cooperare. Entrambi i fattori vanno quindi considerati e attentamente valutati.

In termini metodologici la presente ricerca offre due vantaggi. Da un lato, l'approccio utilizzato rende possibile collegare caratteristiche organizzative di tipo strutturale a variabili relazionali diadiche nell'ambito di una vasta popolazione di organizzazioni; dall'altro, esso permette di considerare simultaneamente e valutare l'impatto di legami competitivi e interazioni collaborative. Poiché la presenza di relazioni esclusivamente competitive o collaborative si riscontra raramente all'interno delle popolazioni organizzative (Pfeffer e Salancik, 1978; Astley, 1984), l'adozione di strumenti di analisi in grado di descrivere in modo appropriato entrambe le tipologie di interdipendenze appare fondamentale al fine di fornire un quadro sempre più realistico dei fenomeni organizzativi.

Lo studio presenta alcuni principali limiti. Un primo limite è legato all'ampiezza del campione preso a riferimento per l'analisi, che include gli ospedali pubblici e i privati accreditati appartenenti al solo servizio sanitario della regione Lazio. Sarebbe utile, ad esempio, estendere l'analisi e confrontare i risultati ottenuti con quelli relativi ad un sistema regionale diverso o includere nell'analisi anche le strutture private, che non partecipano al servizio sanitario nazionale. Il secondo limite è connesso all'analisi quantitativa sviluppata. In effetti, il modello di regressione permette di osservare che la competizione non necessariamente preclude lo scambio interorganizzativo e che anzi, entro certi limiti, essa agisce positivamente sulla propensione delle organizzazioni a collaborare. Questi risultati derivano tuttavia da un'analisi di tipo cross-sectional condotta su dati che, poiché riferiti ad un solo istante temporale, non permettono di investigare nel tempo i rapporti di causalità né tantomeno l'andamento e la forma della relazione che lega competizione e collaborazione. Un'analisi longitudinale che osservi le dinamiche cooperative e competitive tra gli ospedali nel tempo appare opportuna per corroborare i risultati ottenuti nel presente lavoro. Infine, è importante sottolineare che esistono spiegazioni ulteriori, rispetto a quelle che sono state testate con la presente analisi, con riferimento al fenomeno della collaborazione tra ospedali. Tra queste, ad esempio, la fiducia reciproca e i rapporti interpersonali tra medici.

Maggiore competizione per migliorare l'efficienza o più forte integrazione in nome di obiettivi di maggiore efficacia e di equità? La risposta a questa domanda può essere fornita solo attraverso analisi empiriche sull' argomento. La 
speranza è che questo lavoro fornisca lo spunto per un dibattito rinnovato sul legame tra collaborazione e competizione, specie in settori incerti e complessi come quello della sanità, in cui regole istituzionali e tecniche convivono e generano una continua e imprescindibile tensione tra orientamenti all'efficienza e all'equità.

\section{Riferimenti bibliografici}

Aiken, M., Hage, J. (1968), "Organizational interdependence and intraorganizational structure", American Sociological Review, 33: 912-930.

Astley, W.G. (1984), "Toward an appreciation of collective strategy", Academy of Management Review, 9: 526-535.

Barley, S.R., Freeman, J., Hybels, R.C. (1992), "Strategic alliances in commercial biotechnology", in Nohria N., Eccles R. (Eds.), Networks and Organizations: Structure, Form and Action: 31 1-347. Boston: Harvard Business School Press.

Barney, J.B. (1991), "Firm resources and sustained competitive advantage", Journal of Management, 17: 99-120.

Barney, J.B., Hoskisson, R.E., (1990), "Strategic groups: untested assertions and research proposals", Managerial and Decisions Economies, 11: 187-198.

Barnett, W.P. (1993), "Strategic deterrence among multipoint competitors", Industrial and Corporate Change, 2: 249-278.

Barretta, A. (2007), "Il dualismo competizione/cooperazione fra aziende sanitarie pubbliche: un' analisi esplorativa", Mecosan, 61: 49-68.

Baum, J., Korn, H. (1996), "Competitive dynamics of interfirm rivalry", Academy of Management Journal, 39: 255-291.

Bengtsson, M., Powell, W.W. (2004), "Introduction: new perspectives on competition and cooperation", Scandinavian Journal of Management, 20: 1-8.

Bengtsson, M., Kock, S., (2000), "Coopetition" in business networks - to cooperate and compete simultaneously", Industrial Marketing Management, 29: 411-426.

Boeker, W., Goodstein, J., Stephan, H., Murmann, J.P. (1997), "Competition in a multimarket environment: The case of market exit", Organization Science, 8: 126-142.

Borgatti, S.P., Everett, M.G., Freeman, L.C. (2002), Ucinet for Windows: Software for Social Network Analysis. Harvard: Analytic Technologies.

Burns, L.R., Thorpe, D.P.(1993), "Trends and models in physician-hospital organizations", Health Care Management Review, 18: 7-20.

Burt, R.S. (1992), Structural Holes: The Social Structure of Competition. Cambridge, MA: Harvard University Press.

Burt, R.S., Talmud, I. (1993), "Market niche", Social Networks, 15: 133-149.

Butts, C.T., Carley, K.M. (2001), Multivariate Methods for Inter-Structural Analysis. CASOS Working Paper, http://www.casos.ece.cmu.edu/working_papers.html, Carnegie Mellon University.

Callon, M. (1998), "Introduction: The embeddedness of economic markets in economics", in Callon M. (ed.), The laws of the markets: 1-57. Oxford: Blackwell Publishers.

Cohen, W., Levinthal, D., (1990), “Absorptive capacity: a new perspective on learning and innovation”, Administrative Science Quarterly, 35: 128-152.

DiMaggio, P., Powell, W. (1983), "The iron cage revisited: Institutional isomorphism and collective rationality", American Sociological Review, 48: 147-160. 
Dobrev, S., Kim, T-Y. (2006), "Positioning among organizations in a population: Moves between market segments and the evolution of industry structure", Administrative Science Quarterly, 51: 230-261.

Dobrev, S. D., Kim, T.-Y., Hannan, M.T. (2001), "Dynamics of niche width and resource partitioning", American Journal of Sociology, 106: 1299-1337.

Edwards, C.D. (1995), "Conglomerate Bigness as a Source of Power", in Mitchell W.C. (ed.), Business Concentration and Price Policy. Princeton, NJ: Princeton University Press.

Ellinger, R. (1977), "Industrial location behaviour and spatial evolution", Journal of Industrial Economics, 25: 295-312.

Freeman, J., Hannan, M.T. (1983), "Niche Width and the Dynamics of Organizational Populations", American Journal of Sociology, 88: 1116-1145.

Gimeno, J., Woo, C. (1996), "Hypercompetition in a multimarket environment: the role of strategic similarity and multimarket contact in competitive descalation", Organization Science, 7: 322-341.

Gimeno, J., Woo, C. (1999), "Multimarket contact, economies of scope, and firm performance", Academy of Management Journal, 42: 239-259.

Granovetter, M. (1985), "Economic Action and Social Structure: the Problem of Embeddedness", American Journal of Sociology, 91: 481-493.

Greve, H.R. (2006), "The intent and extent of multimarket contact", Strategic Organization, 4: 249-274.

Hannan, M.T., Freeman, J.H. (1977), "The population ecology of organizations", American Journal of Sociology, 83: 929-984.

Hannan, M.T., Freeman, J.H. (1989), Organizational Ecology. Cambridge, MA: Harvard University Press.

Hughes, K., Oughton, C. (1993), "Diversification, multi-market contact and profitability", Economica, 60: 203-224.

Kock, S., (1991), A strategic process for gaining external resources through long-lasting relationships - Examples from two Finnish and two Swedish firms, Multipint, Helsingfors.

Kogut, B., Shan, W., Walker, G. (1992), "Competitive cooperation in biotechnology: Learning through networks?", in Nohria N., Eccles R. (eds.), Networks and Organizations: Structure, Form and Action: 348-365, Boston: Harvard Business School Press.

Krackhardt, D. (1988), "Predicting with networks: Nonparametric multiple regression analysis of dyadic data", Social Networks, 10: 359-381.

Krackhardt, D. (1987), "QAP Partialling as a test of spuriousness", Social Networks, 9: 171-186.

Lega, F., De Pietro, C. (2005), "Converging patterns in hospital organization: beyond the professional bureaucracy", Health Policy, 74: 261-281.

Levine, S., White, P.E. (1961), "Exchange as a Conceptual Framework for the Study of Interorganizational Relationships", Administrative Science Quarterly, 5: 583601.

Mapelli, V. (2007), I sistemi di governance dei Servizi sanitari regionali, Formez, Quaderni Formez, 57.

Meyer, J.W., Rowan, B. (1977), "Institutional organizations: Formal structure as myth and ceremony", American Journal of Sociology, 83: 340-363.

Oliver, A. (2004), "On the duality of competition and collaboration: network-based knowledge relations in the biotecnology industry", Scandinavian Journal of Management, 20: 151-171. 
Oliver, C. (1990), "Determinants of interorganizational relationships: integration and future directions", Academy of Management Review, 15: 241-265.

Pfeffer, J., Salancik G.R. (1978), The External Control of Organizations: A Resource Dependence Perspective, New York, NY: Harper and Row.

Plochg, T., Delnoij, D.M.J., Hoogedoorn, N.P.C., Klazinga, N.S. (2006), “Collaborating while competing? The sustainability of community-based integrated care initiatives through a health partnership", BMC Health Services Research, 6: 1-14.

Podolny, J.M. (1994), "Market uncertainty and the social character of economic exchange", Administrative Science Quarterly, 39: 458-483.

Popielarz, P., McPherson, J. (1995), "On the edge or in between: niche position, niche overlap, and the duration of voluntary association memberships", American Journal of Sociology, 101: 698-720.

Powell, W.W., Koput, K.W., Smith-Doerr, L. (1996), "Interorganizational collaboration and the locus of innovation: Networks of learning in biotechnology", Administrative Science Quarterly, 41: 116-145.

Powell, W.W. (1990), "Neither market nor hierarchy: network form of organization", Research in Organization Behavior, 12: 295-336.

Provan, K.G., Milward, H.B. (1995), “A Preliminary Theory of Interorganizational Network effectiveness: A Comparative Study of Four Community Mental Health Systems", Administrative Science Quarterly, 40: 1-33.

Richardson, G.B. (1972), "The organization of industry", Economic Journal, 82: 883497.

Scott, J.T. (2001), "Designing multimarket-contact hypothesis tests: Patent citations and multimarket contact in the chemicals industry", Advances in Strategic Management, 18.

Shenhar, A.J. (2001), "One size does not fit all projects: Exploring classical contingency domains", Management Science, 47: 394-414.

Sohn, M-W. (2001), "Distance and cosine measures of niche overlap", Social Networks, 23: 141-165.

Sohn, M-W. (2002), "A Relational Approach to Measuring Competition Among Hospitals", Health Services Research, 37: 457-482.

Teece, D.J. (1990), "Contributions and impediments of economic analysis to the study of strategic management", in Frederickson J.W. (ed.), Perspectives on Strategic Management, 39-80, New York: Harper and Row.

Uzzi, B. (1997), "Social structure and competition in interfirm networks: The paradox of embeddedness", Administrative Science Quarterly, 42: 35-67.

Uzzi, B. (1996), "The sources and consequences of embeddedness for the economic performance of organizations: The network effect", American Sociological Review, 61: 674-698.

Williamson, O.E. (1985), The economic institutions of capitalism: Firms, markets, relational contracting, New York, NY: Free Press. 